(C) The Author(s), 2021. Published by Cambridge University Press on behalf of The Nutrition Society. This is an Open Access article, distributed under the terms of the Creative Commons Attribution licence (http://creativecommons.org/licenses/by/4.0/), which permits unrestricted re-use, distribution, and reproduction in any medium, provided the original work is properly cited

\title{
The contribution of provitamin A biofortified cassava to vitamin A intake in Nigerian pre-schoolchildren
}

\author{
Ibukun Afolami ${ }^{1,2}$, Folake Samuel ${ }^{2}$, Karin Borgonjen-van den Berg ${ }^{1}$, Martin N. Mwangi ${ }^{1}$, \\ Olatundun Kalejaiye ${ }^{3}$, Rasaki A. Sanusi ${ }^{2}$, Linda Ayu Rizka Putri ${ }^{1}$, Francesca Brivio ${ }^{1}$, Inge D. Brouwer ${ }^{1}$ and \\ Alida Melse-Boonstra ${ }^{1 *}$ \\ ${ }^{1}$ Division of Human Nutrition and Health, Wageningen University \& Research, Wageningen, the Netherlands \\ ${ }^{2}$ Department of Human Nutrition, College of Medicine, University of Ibadan, Ibadan, Nigeria \\ ${ }^{3}$ HarvestPlus Nigeria, c/o International Institute of Tropical Agriculture (IITA), Ibadan, Nigeria
}

(Submitted 8 August 2020 - Final revision received 4 December 2020 - Accepted 28 December 2020 - First published online 8 January 2021)

Abstract

Biofortified yellow cassava has been developed to alleviate vitamin A deficiency. We examined the potential contribution of yellow cassava to total retinol activity equivalent (RAE) intake if replacing white by yellow cassava among pre-school Nigerian children. Dietary intake was assessed as part of a randomised controlled trial. Pre-schoolchildren ( $n$ 176) were randomly assigned to receive either white cassava (WC) or yellow cassava (YC) for 17 weeks. Dietary intake assessments were conducted during the intervention and 1 month after, when children had resumed their habitual diet. Differences in RAE intake between groups and time points were compared using a linear mixed model regression analysis. During intervention, median RAE intake was $536 \mu \mathrm{g} / \mathrm{d}$ in the YC group and $301 \mu \mathrm{g} / \mathrm{d}$ in the WC group $(P<0 \cdot 0001)$. YC contributed approximately $40 \%$ to total RAE intake. Of the children, $9 \%$ in the YC group and $29 \%$ in the WC group had RAE intake below the Estimated Average Requirement. After intervention, median RAE intake was $300 \mu \mathrm{g} / \mathrm{d}$ and did not differ between intervention groups $(P=0 \cdot 5)$. The interaction effect of group and time showed a $37 \%$ decrease in RAE intake in the YC group after the intervention $(\operatorname{Exp}(\beta)=0 \cdot 63 ; 95 \% \mathrm{CI} 0 \cdot 56,0 \cdot 72)$. If WC was replaced by YC after intervention, the potential contribution of YC to total RAE intake was estimated to be approximately $32 \%$. YC increased total RAE intake and showed a substantially lower inadequacy of intake. It is therefore recommended as a good source of provitamin A in cassava-consuming regions.

Key words: Biofortification: Yellow cassava: Vitamin A: Nigerian children

Vitamin A deficiency (VAD) is still a public health problem in many low-to-middle income countries, affecting approximately 190 million pre-school age children, which corresponds to about $33 \%$ of the children in this age group globally ${ }^{(1)}$. In 2013, the highest prevalence of VAD was in south Asia and sub-Saharan Africa with prevalence of $44 \%$ and $48 \%$, respectively ${ }^{(2)}$. VAD impairs various physiological functions and, as a result, can pose serious health challenges to infants, children and pregnant women $^{(3)}$. Approximately 94500 deaths from diarrhoea and 11200 deaths from measles worldwide were attributed to VAD in 2013, which accounted for $1.7 \%$ of all deaths in children under 5 years in low-to-middle income countries ${ }^{(1)}$.

Nigeria is among the countries with the highest prevalence of VAD in Africa. Based on the available data from the only nationally representative food consumption and nutrient survey
(2004), $29 \cdot 5 \%$ of children under 5 years old were classified as vitamin A deficient, with serum retinol concentrations below $0.7 \mu \mathrm{mol} / \mathrm{l}^{(4)}$, which is attributed to inadequate dietary intake. This high prevalence, which was regarded as a public health problem, led to the implementation of several strategies such as vitamin A supplementation and food fortification to reduce VAD prevalence ${ }^{(5)}$. Over the years, the coverage of vitamin A supplementation has increased in many low- to middle-income countries including Nigeria; however, the impact of vitamin A supplementation on the reduction of VAD has been at a very slow rate ${ }^{(6)}$.

A food-based approach is a more sustainable approach to attaining micronutrient adequacy compared with other approaches ${ }^{(7)}$. Biofortification has emerged as a complementary strategy to meet the micronutrient needs of vulnerable

Abbreviations: EAR, estimated average requirement; FCT, food composition table; RAE, retinol activity equivalent; RCT, randomised controlled trial; RPO, red palm oil; VAD, vitamin A deficiency.

* Corresponding author: Dr Alida Melse-Boonstra, email alida.melse@wur.nl 
populations $^{(8)}$. Specifically, several staples from sub-Saharan Africa and south Asia have been biofortified with provitamin A, with the aim of complementing other vitamin A interventions ${ }^{(9)}$. Two randomised controlled trials (RCT) have demonstrated the efficacy of provitamin A biofortified crops in increasing serum retinol ${ }^{(10)}$ and total body retinol pools ${ }^{(11,12)}$ in children. Yellow cassava, a biofortified variety of the traditional white cassava, is a root and tuber crop, largely consumed in Nigeria. There are currently six varieties of yellow cassava available in Nigeria. These varieties are generally resistant to many pests and diseases, have high yields and can produce up to $15 \mu \mathrm{g} / \mathrm{g}$ of $\beta$-carotene ${ }^{(13)}$. However, till now, no study has considered the contribution of yellow cassava to the usual intake of vitamin A in a natural setting, outside an experimental study set-up.

This study therefore aimed at quantifying the contribution of biofortified (yellow) cassava to vitamin A intake of pre-schoolchildren in a rural Nigerian community where cassava is widely consumed as a common staple, both under experimental and free-living conditions.

\section{Subjects and methods}

\section{Study design and participants}

Dietary assessment was conducted in 2016 as part of a RCT aimed at assessing the efficacy of biofortified cassava varieties on serum vitamin A concentrations in pre-schoolchildren, aged 3-5 years old in three adjacent communities: Telemu, Ilemowu and Asamu Osun state, Nigeria. The study was conducted according to the guidelines laid down in the Declaration of Helsinki, and all procedures involving human subjects were approved by the University of Ibadan/University College Hospital Ethical Review Committee, as well as a positive advice from the Medical Research Ethics Committee, Wageningen University and Research, Wageningen, the Netherlands. State Government approval was also obtained from the Osun State Ministry of Health. The study is registered in clinicaltrial.gov (ID NCT02627222). A total of 176 children were recruited into the RCT including dietary assessments. At least one participant was recruited from the twenty compounds identified in the communities, based on the following criteria: (1) their willingness to participate; (2) no visible sign of sickness; (3) older than 3 years and <5 years before study commencement; (4) plasma $\mathrm{Hb}$ $\geq 70 \mathrm{~g} / \mathrm{l}$ and (5) ability to consume $\geq 80 \%$ of the pre-established age-specific target meal portion for the RCT. Exclusion criteria included (1) severe anaemia $(\mathrm{Hb}<70 \mathrm{~g} / \mathrm{l}) /$ symptomatic malaria or infectious diseases; (2) unwillingness to participate or no informed consent; (3) absenteeism (i.e. not meeting up to $20 \%$ of feeding sessions); (4) inability to consume the required amount of cassava (i.e. $\geq 80 \%$ of the age-specified amount) and (5) history of food allergy.

A pre-school was established specifically for the purpose of the intervention, where students were taught and fed between 08.00 and 14.00 hours. Children whose parents/guardian had signed a written informed consent and who met the conditions for the inclusion criteria were registered in the pre-school, where they were fed breakfast and lunch, $6 \mathrm{~d} /$ week, for a period of
17 weeks. The participants were divided into two groups: the experimental group ( $n$ 88) and the control group ( $n$ 88). The experimental group consumed foods prepared with yellow cassava (yellow cassava eba, yellow cassava garri, yellow cassava moinmoin, complemented with okra or ewedu soup), while the control group consumed the same foods prepared with white cassava. Children in the experimental and control groups were physically separated to prevent crossing-over. The children resumed school at 08.00 hours. Standardised recipes were developed for all foods consumed in the pre-school before the commencement of the intervention.

\section{Dietary intake assessments}

No dietary assessment was conducted at baseline. The first round of dietary assessment was conducted in the 15th week of the study period ( $n$ 162) to estimate dietary intake of the experimental and control groups both within the pre-school and at home. A second round of dietary assessment ( $n$ 158) was conducted 1 month after the intervention, to measure the intake of the study population after they returned to their habitual diets. In each round, repeated recalls were conducted for approximately $30 \%(n 45)$ of the children, to account for day-to-day variation and compute adjusted nutrient intake.

Within the pre-school, intervention foods were prepared in a central kitchen by trained cooks. All the ingredients used in cooking were weighed before cooking. Weights and total volume of dishes cooked were recorded daily and entered into a database. In the pre-school, trained research assistants weighed individual food portions before serving, using a $0 \cdot 1$ precision scale (Kern EMB 5.2K1). The same scales were used throughout the study period. All plates and cups were properly labelled with each participant's ID number. After the meal, leftovers were recorded and entered the same day into a central database.

Dietary intake for foods consumed outside the pre-school was assessed using the quantitative interactive multi-pass 24-h recall method ${ }^{(14,15)}$ on evenly divided days over the week. Repeated recalls were conducted on non-consecutive days and distributed equally across the days of the week. Participants for repeated recalls were randomly selected from both experimental and control groups. Participants' dietary consumption was provided by parents (mother or father) or caregivers, who were directly responsible for children's diets. Dietary assessment was conducted at participant's residence by local research assistants, who spoke the local language and had been trained shortly before the time of the dietary assessment.

During the interview, each parent/caregiver was asked to mention all foods and beverages consumed by their child (from waking up) on the day before the interview, until waking up on the interview day. Detailed information on food ingredients, including preparation/processing methods, total amount prepared, portion consumed by the child and amount of leftovers, were also collected during the recall session. Parents/caregivers were asked to demonstrate the procedures followed while preparing the recalled meals, using when possible, the same ingredients and household utensils (i.e. cups, bowls and spoons). From these demonstrations, the amount of each ingredient in 
each meal consumed was estimated by direct weighing on a digital scale (Kern EMB 5.2K1).

\section{Conversion factors}

In households where the ingredients used for cooking were not available at the time of the recall, the volume or weight of comparable ingredients with similar texture was used as alternatives (i.e. proxy ingredients) during the demonstration, to estimate amount consumed (e.g. yam flour as an alternative to cassava flour, garri as an alternative to rice, etc.). After the recall, the actual ingredients were purchased from the community markets, and conversion factors (i.e. weight-to-weight or volumeto-weight) were computed, to calculate the actual amount of the recalled ingredient. In some households, some of the recalled meals were bought as ready-to-eat dishes from local food vendors. In other households, some of the ingredients used to prepare a particular meal were purchased from the community markets. In both cases, price-to-weight conversion factors were used to estimate the amount of ingredients consumed.

Most of the dishes reported during the recall session were prepared for the entire household, from which the child consumed only a portion. We therefore estimated the total volume of dish cooked and the proportion consumed by the child using the method described by Gibson \& Ferguson ${ }^{(14)}$. Market surveys were conducted during and after the intervention to obtain all conversion factors. The market survey was conducted within 3 weeks after each 24-h recall data collection, to avoid the effect of price fluctuations on conversion factors. During the market surveys, food items or ingredients were purchased in the local currency (naira), from three different shops or vendors where community members frequently purchased their foods. The food items or ingredients were subsequently weighed with and without waste and then averaged. Price-to-weight conversion factors were generated for the edible portion of the food or ingredient, by calculating the amount in the local currency corresponding to $1 \mathrm{~g}$ of the food or ingredient. Weight-to-weight conversion factors were generated by calculating the amount of proxy ingredient corresponding to $1 \mathrm{~g}$ of actual ingredient. Volume-toweight conversion factors were generated by calculating the volume of liquid/semi-solid food or ingredient corresponding to $1 \mathrm{~g}$ of the food or ingredient. During recall, it was noted that some dishes were prepared by combining various food items for a total price, for instance, $100 \mathrm{~g}$ rice $+50 \mathrm{~g}$ beans $+20 \mathrm{~g}$ stew $=\mathrm{N} 50$. In such cases, the proportion of each ingredient within the dish was calculated, so that the weight of each food item could be retrieved separately

\section{Standard recipes}

During the 24-h dietary recall, the details of some food ingredients consumed by children could not be obtained because the dishes were bought from a food vendor or the respondent could not remember the ingredients in the dish. To address these issues, standard recipes were developed to estimate the amount of the ingredient consumed. To develop the standard recipes, three randomly selected volunteer mothers, whose children were participating in the study, were invited and asked to cook the specific dishes, using the same cooking methods employed at home. The different ingredients used in the preparation of the dishes were then recorded and weighed by research assistants while cooking was ongoing. Similarly, standard recipes were collected from random stalls and vendors within the community. In all cases, the edible portions of cleaned ingredients and the total volume of dish cooked were noted.

\section{Carotenoid analysis of food samples}

During the entire study, samples of intervention meals, as consumed, were randomly collected twice weekly for 17 weeks, into opaque containers and homogenised with $5 \mathrm{ml}$ butylhydroquinone (a preservative). Food samples were subsequently stored at $-20^{\circ} \mathrm{C}$ at the field site and later shipped to Wageningen University and Research, where samples were stored at $-80^{\circ} \mathrm{C}$ until analysis. During analysis, the food samples were pooled together as composite samples, representing three different intervention periods. Total $\beta$-carotene in food samples were analysed in duplicate using the HPLC (Thermo Scientific Accela LC system; Thermo Fisher Scientific) and EZCHrom Elite version 3.2.2 SP2 software (Argilent Technologies). The details of the extraction and HPLC analysis have been described elsewhere ${ }^{(16)}$.

\section{$\beta$-Carotene retention in commonly consumed palm oil soups}

$\beta$-Carotene retention in two commonly consumed palm oilbased soups was calculated experimentally. Through the careful observation of volunteer mothers invited to cook these soups, a recipe detailing the average amount of all ingredients in the soup was developed and standardised, taking note of the average cooking time and bleaching temperatures for palm oil. The total $\beta$-carotene content of all raw ingredients was then calculated using a food composition table $(\mathrm{FCT})^{(17)}$, by summing the $\beta$-carotene content of individual raw ingredients, including raw palm oil, which was obtained from the same community. In addition, the $\beta$-carotene content of raw palm oil was analysed. Using the standardised recipes, dishes of stew and egusi soups were prepared experimentally using either raw palm oil or standard zero carotenoid vegetable oil as ingredient. The quantity and type of all ingredients in the analysed soups were identical, and the only difference was the substitution of red palm oil (RPO) with zero carotenoid vegetable oil. Yield factors were calculated for both soups immediately after cooking, and the soups were analysed for total $\beta$-carotene content in the form normally consumed. The true retention factor of $\beta$-carotene $(\beta c a r)$ from the palm oil soups was estimated using the formula:

True retention of $\beta c a r=\frac{\left([\beta c a r]_{\text {palm oil-cooked }}-[\beta c a r]_{\text {veg oil-cooked }}\right)}{\left([\beta c a r]_{\text {palm oil-raw }}\right)} \times$ yield factor

where $[\beta \text { car }]_{\text {palm oil-cooked }}=\beta$-carotene content per $\mathrm{g}$ of cooked soup with palm oil; $[\beta c a r]_{\text {veg oil-cooked }}=\beta$-carotene content per $\mathrm{g}$ of cooked soup with regular vegetable oil; and $[\beta c a r]_{\text {palm oil-raw }}=$ $\beta$-carotene content per $g$ of palm oil in raw soup ${ }^{(18)}$. 


\section{Nutrient intake estimation}

To estimate nutrient intakes, a FCT was compiled for this study based on the Nutrient Composition of Commonly Eaten Foods in Nigeria - raw, processed and prepared ${ }^{(19)}$. The FCT was supplemented with nutrient composition from foods in other FCT in descending order of priority: West African Food Composition Table ${ }^{(17)}$; Condensed Food Composition Table for South Africa ${ }^{(20)}$; United States Department of Agriculture National Nutrient Database for Standard Reference, release $28^{(21)}$ and International Minilist ${ }^{(22)}$.

In the absence of nutrient information for particular foods, nutrient composition was obtained from nutrition labels (for packaged foods) or from published scientific literature. A bioequivalence factor of $7: 1^{(23)}$ was applied to convert $\beta$-carotene to retinol activity equivalent (RAE) from cassava. For all other foods, a bioequivalence factor of 12:1 was applied ${ }^{(24)}$. Appropriate retention factors were applied to adjust for nutrient loss during preparation ${ }^{(18)}$. Compl-Eat software (version 1.0, Wageningen University and Research) was used in the overall computation of nutrient intake ${ }^{(25)}$. Vitamin A intake was expressed as RAE in $\mu \mathrm{g} /$ child per $\mathrm{d}$. The average RAE intake was compared with the estimated average requirement (EAR) to determine the adequacy of vitamin A intake using age-specific cut-off points provided by the Institute of Medicine ${ }^{(24)}$. Estimations of RAE and percentage below EAR during the second round of dietary intake assessment were simulated, based on the assumption that the white cassava foods consumed during this time were replaced by yellow cassava foods. Total energy intake was expressed as $\mathrm{kJ} /$ child per $\mathrm{d}$. Total energy intake was assessed by comparing total daily energy with the Estimated Energy Requirement as established by Institute of Medicine, which considers the sex, age, height, weight and physical activity levels. Based on the observation of the daily routine of the children, a physical activity level of 1.16 (lowactive physical activity level) was used for all children to estimate their energy requirement ${ }^{(24)}$.

\section{Anthropometric measurement}

Height and weight measurements were collected at baseline and endline, using a combined anthropometric 0.2 precision scale and stadiometer (Seca model no. 887 7021094), respectively. Anthropometric parameters (height-for-age, weight-for-age and weight-for-height $z$-scores) were analysed with Anthro (WHO version 3.2.2).

\section{Statistical analysis and sample size}

The statistical analysis was carried out using Stata 13.0. The distribution of all variables used in the analyses was inspected for normality by combining QQ plots and visual inspections. Nonnormal variables were log-transformed. All outliers were retained in the data because none of the estimated intake was considered implausible. Repeated recalls during each round of dietary intake assessment were analysed using ANOVA to estimate the magnitude of the within-person variance and to adjust for day-to-day variation. Following this procedure, adjusted intake distributions were calculated for RAE and energy intake, using the method described by the National Research Council $^{(26)}$. A linear mixed regression method was used to estimate the difference $(\exp \beta)$ in RAE intake between the experimental and control groups based on the first and second rounds of dietary intake assessments. This model is advantageous because of its robustness in handling within-subject correlations resulting from repeated measurements. In fitting the model, log-transformed vitamin A intake was treated as the response variable and the intervention group was included as a fixed effect. Correlation between the two time points of dietary intake assessment was accounted for, by including the random intercept of the subject (ID) in the model. Unstructured covariance with restricted maximum likelihood estimation was used to estimate the variance component. Tukey-Kramer was applied to make multiple comparisons (interactions) of least square means among groups and time points. We adjusted for the confounding effect of age, sex and total energy intake by including these variables in the models. The best model was chosen by comparing the lowest Akaike information criterion. Wilcoxon non-parametric statistic was used to test for the difference in median energy and RAE between participants of different age categories

Based on the projected daily intake of yellow and white cassava, we expected to detect a difference of at least $200 \mu \mathrm{g}$ in the mean RAE daily intake between the yellow and white cassava groups. To this effect, a minimum sample size of 112 participants ( $n 56$ per group) was required, assuming a $15 \%$ attrition rate, an $\alpha$ and $\beta$ risk of 0.05 and $0 \cdot 20$, respectively. This was based on a SD of $318 \mu \mathrm{g}$ as reported in a study conducted in the same region ${ }^{(27)}$.

\section{Results}

\section{Characteristics of the study population}

A total of 162 children participated in the first round of the dietary intake assessment (Table 1), while 158 children participated in the second round (Table 2). The mean age of children was $4 \cdot 1$ (sD 1.0) years. Of the children, $58 \%$ ( $n$ 94) of the children were males (Table 1).

\section{Energy intake and vitamin A intake}

The energy and vitamin A intakes during and after the intervention (i.e. in the first and second rounds of dietary intake assessment) are presented in Table 2 . In the first round of dietary intake assessment, overall median energy intake was $6627 \mathrm{~kJ} / \mathrm{d}$, while in the second round, median energy intake was $6071 \mathrm{~kJ} / \mathrm{d}$ $(P<0 \cdot 001)$. There was no difference in energy intake between boys and girls $(P=0 \cdot 24)$. Over $80 \%$ of the children had adequate energy intake during and after the intervention.

During the intervention, median RAE intake was $536 \mu \mathrm{g} / \mathrm{d}$ and $301 \mu \mathrm{g} / \mathrm{d}$ in the yellow cassava and white cassava groups, respectively $(P<0 \cdot 0001)$. However, after the intervention, median RAE intake decreased to $257 \mu \mathrm{g} / \mathrm{d}$ in the yellow cassava group and increased to $353 \mu \mathrm{g} / \mathrm{d}$ in the white cassava groups, respectively $(P=0.5)$. In total, $82 \%$ of the children had adequate RAE intake during the intervention, which declined to $56 \%$ after the intervention $(P<0 \cdot 0001)$ (Table 2$)$. There was no significant difference in RAE intake between boys and girls during and after 
Table 1. Characteristics of study participants

(Mean values and standard deviations; numbers and percentages)

\begin{tabular}{|c|c|c|c|c|c|c|c|c|c|c|}
\hline \multirow{2}{*}{ Characteristic } & & & \multicolumn{4}{|c|}{ During intervention } & \multicolumn{4}{|c|}{ After intervention } \\
\hline & \multicolumn{2}{|c|}{$\begin{array}{l}\text { All children } \\
\text { ( } n \text { 162) }\end{array}$} & \multicolumn{2}{|c|}{$\begin{array}{l}\text { Yellow cassava } \\
\qquad(n \text { 82) }\end{array}$} & \multicolumn{2}{|c|}{$\begin{array}{l}\text { White cassava } \\
\qquad(n 80)\end{array}$} & \multicolumn{2}{|c|}{$\begin{array}{l}\text { Yellow cassava } \\
\qquad(n 78)\end{array}$} & \multicolumn{2}{|c|}{$\begin{array}{l}\text { White cassava } \\
\qquad(n 80)\end{array}$} \\
\hline \multicolumn{11}{|l|}{ Female } \\
\hline$n$ & \multicolumn{2}{|c|}{68} & \multicolumn{2}{|c|}{35} & \multicolumn{2}{|c|}{33} & \multicolumn{2}{|c|}{33} & \multicolumn{2}{|c|}{34} \\
\hline$\%$ & \multicolumn{2}{|c|}{42} & \multicolumn{2}{|c|}{43} & \multicolumn{2}{|c|}{41} & \multicolumn{2}{|c|}{42} & \multicolumn{2}{|c|}{43} \\
\hline Weight (kg) & 14.38 & 2.06 & $14 \cdot 38$ & $2 \cdot 13$ & 14.38 & $2 \cdot 0$ & 14.9 & $2 \cdot 11$ & $14 \cdot 8$ & 1.93 \\
\hline Height (cm) & $98 \cdot 17$ & 7.56 & 97.97 & $7 \cdot 65$ & $98 \cdot 36$ & $7 \cdot 52$ & 101 & $7 \cdot 3$ & 101 & $7 \cdot 7$ \\
\hline Weight-for-height $z$-score & -0.44 & 0.84 & -0.52 & 0.84 & -0.35 & 0.83 & -0.72 & 0.80 & -0.57 & 0.96 \\
\hline Weight-for-age $z$-score & -1.08 & 0.96 & -1.05 & 0.93 & $-1 \cdot 10$ & 0.99 & -1.12 & 0.93 & -1.21 & 0.94 \\
\hline Height-for-age $z$-score & $-1 \cdot 29$ & 1.31 & $-1 \cdot 19$ & $1 \cdot 26$ & -1.39 & $1 \cdot 37$ & -1.08 & $1 \cdot 24$ & $-1 \cdot 28$ & 1.36 \\
\hline
\end{tabular}

the intervention ( $P=0.58$ and $P=0.13$, respectively). During the intervention, yellow cassava contributed approximately $40 \%$ to the total RAE intake and the percentage of children with retinol activity intakes below the EAR was 9\% $(n 7)$ and $29 \%(n 23)$ in the yellow and white cassava groups, respectively. After the intervention, the percentage of children with retinol intakes below the EAR increased to $43 \%$. Based on the assumption that consumed white cassava foods were all replaced with yellow cassava, the projected contribution to total RAE intake after intervention was estimated to be approximately $32 \%$ (Table 2 ). Palm oil was the second largest contributor to vitamin A intake. During the intervention, palm oil contributed to approximately $24 \%$ and $21 \%$ of RAE intakes in the yellow and white cassava groups, respectively $(P=0 \cdot 6)$. After the intervention, the contribution of palm oil to RAE intake was approximately $35 \%$.

Retention of $\beta$-carotene from palm oil in the soups is presented in Table 3 . Estimated $\beta$-carotene retention from palm oil in stew and egusi vegetable soups was $6.9 \%$ and $6.6 \%$, respectively.

Table 4 presents the effect of yellow cassava on vitamin A intake after adjusting for energy intake, age and sex. Both treatment group $(P<0.0001)$ and time $(P<0.0001)$ significantly explained differences in RAE intake, and their interaction terms showed statistical significance as well. The main effect of the yellow cassava group $v$. the white cassava group on RAE intake was $\operatorname{Exp}(\beta)=1.57$ (95\% CI 1.43, 1.72), whereas the interaction effect of group and time showed that there was a $37 \%$ decrease in RAE intake in the yellow cassava group after the intervention $(\operatorname{Exp}(\beta)=0.63(95 \%$ CI $0.56,0.72)$. Fig. 1 shows the estimated marginal mean intake of RAE in the yellow cassava and white cassava groups during and after the intervention: during the intervention, the yellow cassava and white cassava groups had mean intakes of $458 \mu \mathrm{g}$ and $292 \mu \mathrm{g}$ RAE, respectively, which declined to $309 \mu \mathrm{g}$ in the yellow cassava group and increased to $307 \mu \mathrm{g}$ in the white cassava group, after the intervention.

\section{Discussion}

This study aimed at estimating the contribution of pro-vitamin A biofortified (yellow) cassava to vitamin A intake in Nigerian pre-schoolchildren, both under experimental and free-living conditions. We found that vitamin A intake was $57 \%$ higher in the yellow cassava group compared with the control group during the RCT, whereas intake declined after the intervention. During the RCT, yellow cassava provided sufficient RAE for nearly all the children (96\%). After the intervention, when children had returned to their habitual diets, replacement of white cassava with yellow cassava was estimated to still be able to provide for $32 \%$ of total vitamin A intake and to reduce inadequacy of intake to $0 \%$.

The goal of pro-vitamin A biofortification programmes is to provide biofortified crops that will contribute at least $50 \%$ of the EAR for provitamin A in target populations ${ }^{(28)}$. Our present findings show that the TMS 07/0593 variety of yellow cassava with approximately $9 \mu \mathrm{g} / \mathrm{g}$ (wet weight) $\beta$-carotene used in this study met the set goal under experimental conditions as well as when simulated under free-living conditions. Since breeding programmes are continuously ongoing, newly released varieties can be expected to perform even better. Our findings are also consistent, although higher than another study by Talsma et al. (2016), who, using a linear programming approach, showed that providing a school lunch with yellow cassava to children $6-12$ years old could cover up to $47 \%$ of the recommended nutrient intake of the schoolchildren $^{(10)}$. Recommended nutrient intake are set as $2 \mathrm{SD}$ above the EAR, hence the lower value in their study as compared with ours. Moreover, the methodological differences between the two studies could also be responsible for the differences in estimates.

An important contributor to vitamin A intake in the children's diets was RPO. Over $80 \%$ of the children consumed RPO at least once per $\mathrm{d}$ in one form or another. A different study around the same location showed that RPO consumption was ubiquitous and that about $43 \%$ of children consumed RPO 6 times/week or more ${ }^{(29)}$. Raw RPO can contain high amounts of $\beta$-carotene and has been reported to range between 37300 and $100060 \mu \mathrm{g} / 100 \mathrm{~g}$ in West African $\operatorname{diets}^{(17)}$. To the best of our knowledge, the present study is the first to provide an estimate on $\beta$-carotene retention in commonly consumed Nigerian soups cooked with RPO, although there are few studies that have reported the total carotenoid and $\beta$-carotene content of these dishes ${ }^{(30-33)}$. However, the average retention of $\beta$-carotene in 
Table 2. Energy and vitamin $A$ intake during and after the intervention (Numbers and percentages; median values and 25th, 75th percentiles)

\begin{tabular}{|c|c|c|c|c|c|c|c|c|c|c|c|c|}
\hline \multirow[b]{3}{*}{ Variables } & \multicolumn{4}{|c|}{ During intervention } & \multicolumn{6}{|c|}{ After intervention } & & \\
\hline & \multicolumn{2}{|c|}{ Yellow cassava } & \multicolumn{2}{|c|}{ White cassava } & \multicolumn{2}{|c|}{ Yellow cassava } & \multicolumn{2}{|c|}{ White cassava } & \multicolumn{2}{|c|}{ Total $(n 158)$} & \multicolumn{2}{|c|}{ Projected intake } \\
\hline & Median & $\begin{array}{l}\text { 25th, } 75 \text { th } \\
\text { percentiles }\end{array}$ & Median & $\begin{array}{l}\text { 25th, } 75 \text { th } \\
\text { percentiles }\end{array}$ & Median & $\begin{array}{l}\text { 25th, } 75 \text { th } \\
\text { percentiles }\end{array}$ & Median & $\begin{array}{l}\text { 25th, } 75 \text { th } \\
\text { percentiles }\end{array}$ & Median & $\begin{array}{l}\text { 25th, } 75 \text { th } \\
\text { percentiles }\end{array}$ & Median & $\begin{array}{l}\text { 25th, } 75 \text { th } \\
\text { percentiles }\end{array}$ \\
\hline \multicolumn{13}{|l|}{ Energy intake } \\
\hline \multicolumn{13}{|l|}{ Low† } \\
\hline$n$ & \multirow{2}{*}{\multicolumn{2}{|c|}{$\begin{array}{l}20 \\
24\end{array}$}} & \multirow{2}{*}{\multicolumn{2}{|c|}{$\begin{array}{l}11 \\
14\end{array}$}} & \multirow{2}{*}{\multicolumn{2}{|c|}{$\begin{array}{l}16 \\
21\end{array}$}} & \multirow{2}{*}{\multicolumn{2}{|c|}{$\begin{array}{l}12 \\
15\end{array}$}} & \multirow{2}{*}{\multicolumn{2}{|c|}{28}} & & \\
\hline$\%$ & & & & & & & & & & 18 & & \\
\hline$<4$ years, $\mathrm{kJ} / \mathrm{d}$ & $6301^{a}$ & $5531-7791$ & $6510^{a}$ & $5184-7719$ & $5987^{a}$ & $5778-6381$ & $6004^{\mathrm{b}}$ & $5832-6268$ & 6000 & $5778-6381$ & & \\
\hline$\geq 4$ years, $\mathrm{kJ} / \mathrm{d}$ & $6527^{a}$ & $5430-7021$ & $6933^{a}$ & $6008-7506$ & $6150^{\mathrm{a}}$ & $5678-6364$ & $6104^{\mathrm{b}}$ & $5870-6372$ & 6113 & $5824-6372$ & & \\
\hline Girls, kJ/d & $6498^{\mathrm{a}}$ & $5627-7050$ & $6933^{a}$ & $6150-7719$ & $6025^{a}$ & $5387-6548$ & $6079^{a}$ & $5983-6309$ & 6079 & $5879-6431$ & & \\
\hline Boys, kJ/d & $6385^{a}$ & 5485-7138 & $6590^{\mathrm{a}}$ & $5845-7502$ & $6075^{a}$ & $5653-6305$ & $6071^{a}$ & $5766-6372$ & 6075 & $5661-6314$ & & \\
\hline \multicolumn{13}{|l|}{$\begin{array}{l}\text { RAE intake } \\
\text { Inadequacy }\end{array}$} \\
\hline$n$ & \multirow{2}{*}{\multicolumn{2}{|c|}{7}} & \multirow{2}{*}{\multicolumn{2}{|c|}{$\begin{array}{l}23 \\
29\end{array}$}} & \multirow{2}{*}{\multicolumn{2}{|c|}{$\begin{array}{l}38 \\
49\end{array}$}} & \multirow{2}{*}{\multicolumn{2}{|c|}{$\begin{array}{l}32 \\
40\end{array}$}} & \multirow{2}{*}{\multicolumn{2}{|c|}{$\begin{array}{l}70 \\
44\end{array}$}} & & \\
\hline$\%$ & & & & & & & & & & & & \\
\hline$<4$ years, $\mu \mathrm{g} / \mathrm{d}$ & $563^{a}$ & $374-623$ & $293^{b}$ & $260-392$ & $249^{b}$ & $178-649$ & $370^{b}$ & $170-622$ & 307 & $177-625$ & 475 & $313-626$ \\
\hline$\geq 4$ years, $\mu \mathrm{g} / \mathrm{d}$ & $469^{a}$ & $328-586$ & $303^{b}$ & $246-388$ & $263^{b}$ & 184-532 & $350^{b}$ & $186-526$ & 292 & $186-526$ & 561 & $358-1017$ \\
\hline Girls, $\mu \mathrm{g} / \mathrm{d}$ & $533^{a}$ & $308-606$ & $310^{b}$ & $260-462$ & $261^{b}$ & $198-528$ & $397^{b}$ & $223-698$ & 373 & $198-584$ & 531 & 383-922 \\
\hline Boys, $\mu \mathrm{g} / \mathrm{d}$ & $542^{a}$ & $350-614$ & $288^{b}$ & $251-363$ & $254^{b}$ & $178-555$ & $300^{b}$ & $162-450$ & 273 & $163-480$ & 475 & $318-743$ \\
\hline
\end{tabular}

RAE, retinol activity equivalents; IOM, Institute of Medicine.

a,b Median values within a row with unlike superscript letters are statistically significantly different. Row letters compare both yellow and white cassava groups during and after the intervention.

* Calculated from post-intervention data at time point 2 based on the assumption that yellow cassava foods replaced all white cassava foods for all children.

†Low energy intake is defined as energy intake less than IOM's recommended age-specific requirement, 
Table 3. Retention of total $\beta$-carotene in red palm oil (RPO)-based soups ${ }^{\star}$

\begin{tabular}{|c|c|c|c|c|c|c|c|}
\hline \multirow[b]{2}{*}{ Dish name } & \multicolumn{3}{|c|}{ Raw ingredients } & \multicolumn{4}{|c|}{ Cooked dish } \\
\hline & Name & Amount (g) & $\beta$-Carotene† $(\mu \mathrm{g})$ & Amount (g) & Yield factor & $\begin{array}{c}\beta \text {-Carotene with RPO } ¥ \\
(\mu \mathrm{g} / 100 \mathrm{~g})\end{array}$ & $\begin{array}{l}\beta \text {-Carotene without RPO† } \\
(\mu \mathrm{g} / 100 \mathrm{~g})\end{array}$ \\
\hline \multirow[t]{9}{*}{ Stew } & Tatase & 69 & 1587 & - & & - & 1746 \\
\hline & Chili pepper & 39 & 250 & - & & - & 244 \\
\hline & Onion & 62 & - & - & & - & - \\
\hline & Tomato & 183 & 1142 & - & & - & 1405 \\
\hline & Palm oil & 200 & 137360 & - & & - & - \\
\hline & Seasoning & 4 & - & - & & - & - \\
\hline & Salt & 2 & - & - & & - & - \\
\hline & Water & 250 & - & - & & - & - \\
\hline & Total & 809 & 140761 & 370 & 0.46 & 5946 & 3395 \\
\hline \multirow[t]{9}{*}{ Egusi soup } & Tatase & 108 & 2484 & & & & 2732 \\
\hline & Chili pepper & 78 & 499 & & & & 488 \\
\hline & Onion & 96 & - & & & & - \\
\hline & Palm oil & 250 & 171700 & & & - & \\
\hline & Amaranthus & 200 & 5780 & & & & 5480 \\
\hline & Melon & 60 & - & & & & - \\
\hline & Salt & 10 & - & & & & - \\
\hline & Water & 550 & - & & & & - \\
\hline & Total & 1352 & 180463 & 935 & 0.69 & 9908 & 8700 \\
\hline
\end{tabular}

* The carotenoid content of RPO is based on analysed value.

† Values are based on the reported values in the West-African Food Composition Table ${ }^{(17)}$. $\ddagger$ Values are based on laboratory analyses of cooked dish.

Table 4. Effect of yellow cassava on vitamin A intake (Mean values and $95 \%$ confidence intervals)

\begin{tabular}{lccc}
\hline Variable & $\beta$ (exp) & $95 \% \mathrm{Cl}$ & $P$ value \\
\hline Intervention group* & 1.57 & $1.43,1.72$ & $<0.0001$ \\
Time point $\dagger$ & 1.06 & $0.97,1.16$ & 0.21 \\
Group $\times$ time & 0.63 & $0.56,0.72$ & $<0.0001$ \\
\hline
\end{tabular}

* Yellow cassava group $v$. white cassava group

† Time point 2 (after intervention) $v$. time point 1 (during intervention).

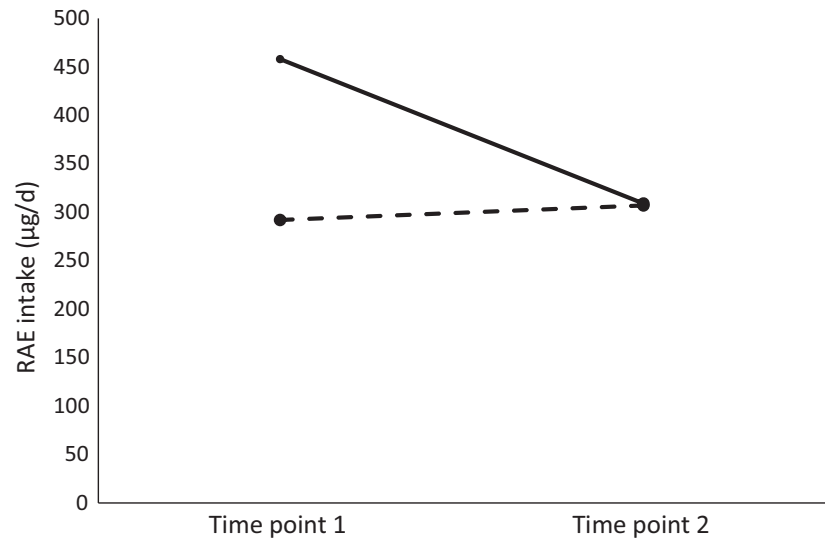

Fig. 1. Estimated marginal mean retinol activity equivalent (RAE) intake in the yellow cassava and white cassava groups. Time point $1=1$ st round of dietary intake assessment (during intervention); time point $2=2$ nd round of dietary intake assessment (after intervention). $\longrightarrow$, Yellow cassava; $\bullet \rightarrow$, white cassava.

RPO estimated from this study may not be applicable to the entire Nigerian population, as cooking methods differ widely across ethnic groups in the country ${ }^{(34)}$.

RPO was mostly used to prepare stews and vegetable soups, which were usually consumed along with a starch-based staple food. A comparison of previous studies on the carotenoid content of egusi vegetable soup showed that $\beta$-carotene varied between $204 \mu \mathrm{g} / 100 \mathrm{~g}^{(35)}$ and $13047 \mu \mathrm{g} / 100 \mathrm{~g}^{(31)}$. This large variation in carotenoid content can be partly explained by differences in cooking methods. For example, we observed that bleaching of RPO prior to cooking was a common practice amongst mothers in the community where our study was conducted. It may therefore be erroneous to apply the $\beta$-carotene concentration values of similar soups, directly from FCT without accounting for cooking time and method, and consequently, without appropriate retention factors for RPO, which is the largest source of carotenoids in these dishes ${ }^{(32,36)}$. We estimated that RPO consumption contributed about $35 \%$ to total RAE intake, which was barely over half of the value reported by De Moura et al., in a study on cassava intake among women and pre-schoolchildren in Akwa-Ibom, southern Nigeria $^{(36)}$. In that study, the contribution of palm oil to daily RAE was about three times higher than the estimated value in the present study. This disparity is probably because authors did not account for the loss of $\beta$-carotene from RPO upon heating since solid data for the $\beta$-carotene content of many Nigerian dishes are lacking. Based on the widespread consumption of RPO in Nigeria, further studies are needed to understand the amount of $\beta$-carotene that is retained from RPO-based foods under different cooking conditions. This will lead to more precise estimations of the contribution of RPO to vitamin A intake. Furthermore, it would also be interesting to explore the possibility of adapting cooking habits such that $\beta$-carotene from $\mathrm{RPO}$ is better retained.

From our study, despite the contribution of RPO, approximately $43 \%$ of the children had inadequate vitamin A intake when consuming their habitual diets (Table 2 ). This clearly suggests that vitamin A intake is still lower than that recommended in this population, a gap which can be filled by promoting 
biofortification and other food-based strategies to improve vitamin A intake.

For the conversion of $\beta$-carotene to retinol, we assumed a bioequivalence factor of 7:1 for biofortified cassava-based foods in the present study. Some studies have shown that the bioequivalence of pro-vitamin A from biofortified cassava can be as high as 4:1 based on experimental studies with single meals under highly controlled conditions in healthy adults ${ }^{(18)}$. We used a more conservative value since the study was conducted in children living in a poor resource setting where malnutrition, intestinal parasite infestation and infectious disease are rife. If we would have used a higher conversion factor, the contribution of biofortified cassava to vitamin A intake would obviously have been even higher than our current estimates.

A very important strength of this study was that we measured nutrient intake during the intervention and afterwards, when the participants had returned to their usual diets. This provided a more accurate estimation of the contribution of yellow cassava to provitamin A intake during the intervention, as well as a realistic estimate of the potential increase in vitamin A intake that would occur if yellow cassava replaced white cassava. Other important strengths were the steps taken to improve the accuracy of nutrient intake estimation. First and foremost, we conducted laboratory analyses of biofortified food samples and commonly consumed RPO-based foods, which provided more reliable nutrient composition data, especially for biofortified foods which were not available in the national food composition database. Second, we developed recipes for all biofortified foods and commonly consumed local soups because standard recipes were unavailable for most of the soups consumed in the community households. Third, we estimated nutrient intake as a sum total of all individual ingredients used in the preparation of a particular dish. This method is deemed to be more reliable especially in rural communities in developing countries where standard recipes may not be available for many dishes ${ }^{(37)}$. Furthermore, we adjusted nutrient and energy intake distribution for day-to-day variation using repeated recalls. This provided a more accurate estimation of usual intake, by partially eliminating day-to-day variation.

This study also had some limitations. First, to estimate the nutrient value of some foods, values from FCT from multiple countries were adopted because the Nigerian Food Composition table did not cover the composition of all foods consumed in the community. A FCT in principle should be country-specific; thus, using FCT from other countries may have also introduced some errors in nutrient intake estimation. In addition, information on fortification was unavailable in most of the FCT consulted. Therefore, the vitamin A content of some fortified flour-based snacks, sugars and oils where proper nutrition labels were absent was not captured, thus contributing to errors. However, we expect that such errors would have been minimal because these food products were minimally consumed. For example, we were unable to compute possible vitamin A intake from possibly fortified flour used as an ingredient in biscuit, in which about $18 \%$ of the children consumed an average of $23 \mathrm{~g}$ during the recall days. Moreover, compliance to mandatory food fortification for sugar and oil has been reported to be $<20 \%$ in Nigeria $^{(38)}$. For our simulation, we assumed that, after intervention, all white cassava in the children's diets would be replaced by biofortified varieties. However, in reality, this may not be the case, as some people may still have a high preference for white cassava. Finally, we did not account for seasonal variation in vitamin A intake. For example, certain pro-vitamin A-rich foods, such as mango, were unavailable during the season the study was conducted. This may also have contributed to errors in estimating the habitual intake of vitamin A as well as the percentage of children below the EAR.

In conclusion, yellow cassava contributed approximately $40 \%$ of the total intake of vitamin $\mathrm{A}$ and has the potential to reduce the percentage of children at risk of inadequate intakes of vitamin A to a low level. Yellow cassava is therefore recommended as a good source of provitamin A in cassava-consuming regions.

\section{Acknowledgements}

The authors specially thank all the community members of Telemu, Ilemowu and Asamu communities for their active and engaging participation in this study. The authors acknowledge specially all the community leaders and their support towards the successful completion of the study. With fond memories, they remember the late Mrs Kehinde Deborah, a dedicated study cook, who passed away shortly after the project.

This study was funded by HarvestPlus, Washington, DC, USA. HarvestPlus had no role in the design, analysis and writing of this article.

The authors' responsibilities: I. A. coordinated the field work, conducted the statistical analysis and prepared the first draft of the manuscript; F. S. provided general management and facilitated field logistic; K. B. supervised and planned the dietary intake assessment; M. N. M. supervised the study and was involved in field administration; O. K. was involved in meal planning and cassava transportation logistics; R. S. was involved in field administration; L. P. was involved in field administration and data collection; F. B. was involved in field administration and data collection; I. B. coordinated dietary intake assessment; A. M.-B. oversaw all aspects of the study and was responsible for the study; all authors contributed to the final manuscript. O. K. is employed by HarvestPlus.

The authors do not have any conflicts of interest.

\section{References}

1. Stevens GA, Bennett JE, Hennocq Q, et al. (2015) Trends and mortality effects of vitamin A deficiency in children in 138 lowincome and middle-income countries between 1991 and 2013: a pooled analysis of population-based surveys. Lancet Glob Health 3, e528-e536.

2. World Health Organization (2009) Global Prevalence of Vitamin A Deficiency in Populations at Risk 1995-2005. Geneva, Switzerland: WHO.

3. McLaren DS \& Kraemer K (2012) Manual on Vitamin A Deficiency Disorders (VADD). Basel, Switzerland: Karger Medical and Scientific Publishers.

4. Maziya-Dixon BB, Akinyele IO, Sanusi RA, et al. (2006) Vitamin A deficiency is prevalent in children less than $5 \mathrm{y}$ of age in Nigeria. J Nutr 136, 2255-2261. 
5. Dalmiya N \& Palmer A (2007) Vitamin A Supplementation: A Decade of Progress. New York: UNICEF.

6. Mason J, Greiner T, Shrimpton R, et al. (2015) Vitamin A policies need rethinking. Int J Epidemiol 44, 283-292.

7. Low JW, Arimond M, Osman N, et al. (2007) A food-based approach introducing orange-fleshed sweet potatoes increased vitamin A intake and serum retinol concentrations in young children in rural mozambique. J Nutr 137, 1320-1327.

8. Nestel P, Bouis HE, Meenakshi JV, et al. (2006) Biofortification of staple food crops. J Nutr 136, 1064-1067.

9. Moura D, Palmer AC, Finkelstein JL, et al. (2014) Are biofortified staple food crops improving vitamin A and iron status in women and children? New evidence from efficacy trials. Adv Nutr 5, 568-570.

10. Talsma EF, Brouwer ID, Verhoef H, et al. (2016) Biofortified yellow cassava and vitamin A status of Kenyan children: a randomized controlled trial. Am J Clin Nutr 103, 258-267.

11. van Jaarsveld PJ, Faber M, Tanumihardjo SA, et al. (2005) $\beta$-Carotene-rich orange-fleshed sweet potato improves the vitamin A status of primary school children assessed with the modified-relative-dose-response test. Am J Clin Nutr 81, 1080-1087.

12. Gannon B, Kaliwile C, Arscott SA, et al. (2014) Biofortified orange maize is as efficacious as a vitamin A supplement in Zambian children even in the presence of high liver reserves of vitamin A: a community-based, randomized placebocontrolled trial. Am J Clin Nutr 100, 1541-1550.

13. Ayetigbo O, Latif S, Abass A, et al. (2018) Comparing characteristics of root, flour and starch of biofortified yellow-flesh and white-flesh cassava variants, and sustainability considerations: a review. Sustainability 10,3089.

14. Gibson RS \& Ferguson EL (2008) An Interactive 24-Hour Recall for Assessing the Adequacy of Iron and Zinc Intakes in Developing Countries. Washington, DC: HarvestPlus.

15. Conway JM, Ingwersen LA, Vinyard BT, et al. (2003) Effectiveness of the US Department of Agriculture 5-step multiple-pass method in assessing food intake in obese and nonobese women. Am J Clin Nutr 77, 1171-1178.

16. Afolami I, Mwangi MN, Samuel F, et al. (2021) Daily consumption of pro-vitamin A biofortified (yellow) cassava improves serum retinol concentrations in preschool children in Nigeria: a randomized controlled trial. Am J Clin Nutr 113, 221-231.

17. Stadlmayr B, Charrondiere U, Enujiugha V, et al. (2012) West African Food Composition Table. Rome: FAO.

18. US Department of Agriculture (2007) Table of Nutrient Retention Factors, release 6.0. https://www.ars.usda.gov/ ARSUserFiles/80400525/Data/retn/retn06.pdf (accessed January 2021).

19. Oguntona B \& Akinyele I (1995) Nutrient Composition of Commonly Eaten Foods in Nigeria: Raw, Processed and Prepared. Ibadan, Nigeria: Food Basket Foundation International.

20. Wolmarans P, Danster N, Dalton A, et al. (2010) Condensed Food Composition Tables for South Africa. Parow Valley, Cape Town: South African Medical Research Council.

21. US Department of Agriculture. National Nutrient Database for Standard Reference, release 28. http://www.ars.usda.gov/ba/ bhnrc/ndl (accessed January 2021).

22. International Food Composition table/database directory. http://www.fao.org/infoods/infoods/tables-and-databases/ en/ (accessed January 2021).
23. Van Loo-Bouwman CA, Naber THJ, et al. (2014) A review of vitamin A equivalency of $\beta$-carotene in various food matrices for human consumption. Br J Nutr 111, 2153-2166.

24. Institute of Medicine (US) Food and Nutrition Board (1998) Dietary Reference Intakes: A Risk Assessment Model for Establishing Upper Intake Levels for Nutrients. Washington, DC: National Academies Press. http://www.ncbi.nlm.nih.gov/ books/NBK45189/ (accessed January 2020).

25. Compleat Nutrition Database. www.compleat.nl (accessed January 2021).

26. National Research Council (1986) Nutrient Adequacy: Assessment Using Food Consumption Surveys. Washington, DC: National Academies Press.

27. Ijarotimi OS (2004) Evaluation of energy and micronutrients intake of Nigerian adolescent females: a case study of institutionalized secondary schools in Akure South Local Government Area, Ondo State, Nigeria. Pak J Nutr 3, 250-253.

28. HarvestPlus, International Center for Tropical Agriculture (CIAT), Cali, Colombia \& Andersson M (2017) Progress update: Crop development of biofortified staple food crops under HarvestPlus. Afr J Food Agric Nutr Dev 17, 11905-11935.

29. Abolurin O, Adegbola A, Oyelami O, et al. (2018) Vitamin A deficiency among under-five Nigerian children with diarrhoea. Afr Health Sci 18, 737.

30. Ene-Obong HN, Sanusi RA, Udenta EA, et al. (2013) Data collection and assessment of commonly consumed foods and recipes in six geo-political zones in Nigeria: important for the development of a National Food Composition Database and Dietary Assessment. Food Chem 140, 539-546.

31. Ajani Sanu R \& Eniola Ade A (2009) Beta carotene content of commonly consumed foods and soups in Nigeria. PakJ Nutr 8, 1512-1516.

32. Alozie YE \& Ene-Obong HN (2018) Recipe standardization, nutrient composition and sensory evaluation of waterleaf (Talinum triangulare) and wild spinach (Gnetum africanum) soup "afang" commonly consumed in South-South Nigeria. Food Chem 238, 65-72.

33. Ayogu R, Edeh R, Madukwe E, et al. (2017) Commonly consumed foods: nutritional quality and contributions to recommended nutrient intakes of schoolchildren in rural Southeastern Nigeria. Food Nutr Bull. 38, 65-77.

34. Davidson GI, Ene-Obong HN \& Chinma CE (2017) Variations in nutrients composition of most commonly consumed cassava (Manihot esculenta) mixed dishes in South-Eastern Nigeria. J Food Qual 2017, 6390592.

35. Okeke EC, Eneobong AO, Ozioko AO, et al. (2009) Nutrient composition of traditional foods and their contribution to energy and nutrient intakes of children and women in rural households in Igbo culture area. Pak J Nutr 8, 304-312.

36. De Moura FF, Moursi M, Lubowa A, et al. (2015) Cassava intake and vitamin A status among women and preschool children in Akwa-Ibom, Nigeria. PLOS ONE 10, e0129436.

37. Gibson RS \& Ferguson EL (2008) An interactive 24-hour recall for assessing the adequacy of iron and zinc intakes in developing countries. Wash DC Harvest. http://www.ifpri.org/sites/ default/files/publications/tech08.pdf (accessed November 2014).

38. Ogunmoyela OA, Adekoyeni O, Aminu F, et al. (2013) A critical evaluation of survey results of vitamin A and Fe levels in the mandatory fortified food vehicles and some selected processed foods in Nigeria. Niger Food J 31, 52-62. 